\title{
Magnetotransport in semiconductors and two-dimensional materials from first principles
}

\author{
Dhruv C. Desai, ${ }^{1}$ Bahdan Zviazhynski $\odot,{ }^{2}$ Jin-Jian Zhou, ${ }^{1}$ and Marco Bernardi $\odot^{1, *}$ \\ ${ }^{1}$ Department of Applied Physics and Materials Science, California Institute of Technology, Pasadena, California 91125, USA \\ ${ }^{2}$ Trinity College, University of Cambridge, Cambridge, CB2 1TQ, United Kingdom
}

(Received 19 January 2021; revised 22 March 2021; accepted 23 March 2021; published 7 April 2021)

\begin{abstract}
We demonstrate a first-principles method to study magnetotransport in materials by solving the Boltzmann transport equation (BTE) in the presence of an external magnetic field. Our approach employs ab initio electronphonon interactions and takes spin-orbit coupling into account. We apply our method to various semiconductors (Si and GaAs) and two-dimensional (2D) materials (graphene) as representative case studies. The magnetoresistance, Hall mobility, and Hall factor in $\mathrm{Si}$ and GaAs are in very good agreement with experiments. In graphene, our method predicts a large magnetoresistance, consistent with experiments. Analysis of the steady-state electron occupations in graphene shows the dominant role of optical phonon scattering and the breaking of the relaxation time approximation. Our paper provides a detailed understanding of the microscopic mechanisms governing magnetotransport coefficients, establishing the BTE in a magnetic field as a broadly applicable first-principles tool to investigate transport in semiconductors and 2D materials.
\end{abstract}

DOI: 10.1103/PhysRevB.103.L161103

\section{INTRODUCTION}

Magnetic fields can strongly influence the electrical properties of materials, with changes quantified by magnetotransport coefficients such as the magnetoresistance (MR), Hall mobility and Hall factor [1,2]. In metals and semiconductors, the change in resistivity with magnetic field is typically small, but in certain semimetals, magnetic heterostructures, and oxides, the effects can be far greater or even dramatic, as in the case of giant and colossal MR [3,4]. Magnetotransport is of practical relevance for various applications, including sensors [5], magnetoresitive RAM, and hard drives [6]. In addition, measurements of the carrier concentration and electrical mobility require knowledge of the Hall factor. Therefore, it is important to understand the physical mechanisms governing magnetotransport and develop methods to accurately predict the MR and Hall factor.

Experimentally, magnetotransport has been studied extensively in metals [2] and simple semiconductors such as $\mathrm{Si}$ [7-12] and GaAs [13-15]. More recently, measurements on two-dimensional materials have shown unconventional behaviors, such as large nonsaturating MR at high fields in graphene [16-18] and $\mathrm{WTe}_{2}$ [19], and various studies have shown an interplay between band-structure topology and magnetotransport, including the chiral anomaly and negative MR in topological semimetals [20-22]. These developments show that magnetotransport is a rapidly growing research arena.

Early attempts to formulate theories of magnetotransport phenomena [23] focused on approximate solutions of the Boltzmann transport equation (BTE) in the relaxation time approximation (RTA) [1]. Subsequent work using parametrized electronic band structures and electron-phonon (e-ph) inter-

*bmarco@caltech.edu actions has shown calculations of the Hall factor in various materials [24,25]. Approaches beyond the RTA have also been proposed, for example, by solving BTE in polar semiconductors in terms of infinite determinants [26] or computing the phonon-limited Hall mobility in Si using deformation potential theory $[27,28]$. These models lack analytic closedform solutions and thus were implemented numerically, highlighting the need for computational approaches to study magnetotransport.

In recent years, density functional theory (DFT) [29] and density functional perturbation theory (DFPT) [30] have enabled $a b$ initio calculations of $e$-ph interactions. The resulting phonon-limited charge transport has been studied in various semiconductors and 2D materials in the framework of the BTE [31-39]. First-principles studies of magnetotransport have lagged behind - the only existing examples are two works by Macheda et al., who investigated an insulator (diamond) [40] and, very recently, the Hall factor in graphene [41] by solving the BTE in a magnetic field, as well as methods employing the Fermi surface topology to investigate magnetotransport [42]. However, first-principles calculations of magnetotransport in semiconductors are still missing and the MR in 2D materials has not yet been computed.

Here we show calculations of the MR, Hall mobility, and Hall factor as a function of temperature and magnetic field, in group-IV and polar semiconductors, focusing on the prototypical cases of $\mathrm{Si}, \mathrm{GaAs}$, and in graphene. Our approach, implemented in our open-source PERTURBO code [43], solves the linearized BTE in a magnetic field using Jacobi iteration (as opposed to conjugate gradient implemented in Ref. [40]) to obtain the conductivity tensor and from it the magnetotransport properties. The calculations employ $a b$ initio $e$-ph interactions and include spin-orbit coupling (SOC), which is particularly important for holes. We evaluate $\mathbf{k}$-space derivatives from a central finite difference approximation [44] and 
obtain the conductivity using tetrahedron integration. To our knowledge, magnetotransport calculations employing such precise numerical techniques and correctly including SOC have not yet been reported. Extensive comparisons with experiments demonstrate the accuracy of our first-principles magnetotransport calculations for semiconductors. Analysis of the relative occupation changes in momentum space shows the dominant role of backscattering due to optical phonons and the breaking of the RTA in graphene. Taken together, our work demonstrates an accurate method to investigate magnetotransport in semiconductors and 2D materials and clarify the underlying microscopic mechanisms.

\section{METHODS}

\section{A. Magnetotransport properties and BTE}

In the presence of small electric (E) and magnetic (B) fields, the current density $\mathbf{J}$ can be written as

$$
J_{i}=\sum_{j=1}^{3} \sigma_{i j}(\mathbf{B}) E_{j},
$$

with the conductivity tensor $\sigma_{i j}$ expanded as [1]

$$
\sigma_{i j}(\mathbf{B})=\sigma_{i j}^{(0)}+\sigma_{i j k}^{(1)} B_{k}+\sigma_{i j k l}^{(2)} B_{k} B_{l}+\ldots,
$$

with implied summations over repeated indices (which correspond to Cartesian components). We write the current in terms of electronic occupations $f_{n \mathbf{k}}$ and band velocities $v_{n \mathbf{k}}(n$ is the band index and $\mathbf{k}$ the crystal momentum of the electronic state),

$$
\mathbf{J}=\frac{-S e}{\mathcal{N}_{\mathbf{k}} \Omega} \sum_{n \mathbf{k}} f_{n \mathbf{k}} \mathbf{v}_{n \mathbf{k}},
$$

where $e$ is the absolute value of the electric charge, $S$ the spin degeneracy, $\mathcal{N}_{\mathbf{k}}$ the number of unit cells, and $\Omega$ their volume. At steady-state, the BTE in the presence of both electric and magnetic fields reads [1]

$$
e \frac{\partial f_{n \mathbf{k}}}{\partial \epsilon_{n \mathbf{k}}} \mathbf{v}_{n \mathbf{k}} \cdot \mathbf{E}+\frac{e}{\hbar}\left(\mathbf{v}_{n \mathbf{k}} \times \mathbf{B}\right) \cdot \nabla_{\mathbf{k}} f_{n \mathbf{k}}+\mathcal{I}^{\mathrm{e}-\mathrm{ph}}\left[f_{n \mathbf{k}}\right]=0,
$$

where $\epsilon_{n \mathbf{k}}$ are electronic energies and the last term includes $e$-ph collision processes consisting of absorption or emission of a phonon [43]. Expanding $f_{n \mathbf{k}}$ to leading order in $\mathbf{E}$, we write $f_{n \mathbf{k}}-f_{n \mathbf{k}}^{0}=-f_{n \mathbf{k}}^{0}\left(1-f_{n \mathbf{k}}^{0}\right) \frac{e \mathbf{E}}{k_{B} T} \cdot \mathbf{F}_{n \mathbf{k}}$ and solve for the unknown occupation changes $\mathbf{F}_{n \mathbf{k}}$ [43]. Factoring out $-e \mathbf{E} f_{n \mathbf{k}}^{0}\left(1-f_{n \mathbf{k}}^{0}\right) / k_{B} T$, we obtain the linearized BTE

$$
\mathbf{v}_{n \mathbf{k}}+\frac{e}{\hbar}\left(\mathbf{v}_{n \mathbf{k}} \times \mathbf{B}\right) \nabla_{\mathbf{k}} \mathbf{F}_{n \mathbf{k}}=\frac{1}{\mathcal{N}_{\mathbf{q}}} \sum_{m, \nu \mathbf{q}} W_{n \mathbf{k}, m \mathbf{k}+\mathbf{q}}^{\nu \mathbf{q}}\left(\mathbf{F}_{n \mathbf{k}}-\mathbf{F}_{m \mathbf{k}+\mathbf{q}}\right),
$$

where $v$ is the phonon mode index, $\mathbf{q}$ the phonon wave vector, and $\mathcal{N}_{\mathbf{q}}$ the number of $\mathbf{q}$ points used in the summation. Here, $W_{n \mathbf{k}, m \mathbf{k}+\mathbf{q}}^{v \mathbf{q}}$ is the scattering rate from $|n \mathbf{k}\rangle$ to $|m \mathbf{k}+\mathbf{q}\rangle$ and takes into account both phonon absorption and emission processes [43]. We solve for $\mathbf{F}_{n \mathbf{k}}$ by rearranging terms in Eq. (5) and using the iterative Jacobi scheme. For each iteration $i$, we get

$$
\begin{aligned}
\mathbf{F}_{n \mathbf{k}}^{(i+1)}= & \mathbf{v}_{n \mathbf{k}} \tau_{n \mathbf{k}}+\frac{\tau_{n \mathbf{k}}}{\mathcal{N}_{\mathbf{q}}} \sum_{m, v \mathbf{q}} W_{n \mathbf{k}, m \mathbf{k}+\mathbf{q}}^{\nu \mathbf{q}} \mathbf{F}_{m \mathbf{k}+\mathbf{q}}^{(i)} \\
& +\frac{e}{\hbar} \tau_{n \mathbf{k}}\left(\mathbf{v}_{n \mathbf{k}} \times \mathbf{B}\right) \nabla_{\mathbf{k}} \mathbf{F}_{n \mathbf{k}}^{(i)},
\end{aligned}
$$

where $\tau_{\mathrm{nk}}$ is the relaxation time. The term containing the gradient in $\mathbf{k}, \nabla_{\mathbf{k}} \mathbf{F}_{n \mathbf{k}}$, is computed using the central finite difference approximation in Ref. [44]. Starting with the RTA solution as the initial guess, $\mathbf{F}_{n \mathbf{k}}=\mathbf{v}_{n \mathbf{k}} \tau_{n \mathbf{k}}$, we evaluate the right-hand side of Eq. (6) to update the solution $\mathbf{F}_{n \mathbf{k}}$, iterating this procedure until convergence.

Expanding $f_{n \mathbf{k}}$ in Eq. (3), we obtain [43]

$$
\sigma_{i j}=\frac{e^{2} S}{\mathcal{N}_{\mathbf{k}} \Omega k_{\mathrm{B}} T} \sum_{n \mathbf{k}} f_{n \mathbf{k}}^{0}\left(1-f_{n \mathbf{k}}^{0}\right)\left(\mathbf{v}_{n \mathbf{k}}\right)_{i}\left(\mathbf{F}_{n \mathbf{k}}\right)_{j} .
$$

We can calculate the magnetotransport coefficients from this conductivity tensor because of its implicit dependence on $\mathbf{B}$ through Eq. (6). The MR can be obtained from the resistivity tensor $\rho(\mathbf{B})=\sigma^{-1}(\mathbf{B})$ using [1]

$$
\operatorname{MR}=\frac{\rho(\mathbf{B})-\rho(0)}{\rho(0)} .
$$

At low fields, the MR is expected to be quadratic in the magnetic field [45]. In most materials, the MR perpendicular to $\mathbf{B}$ (transverse MR) is small and positive-classically, this increase in resistivity can be viewed as a result of the Lorentz force deviating charge carriers from their initial trajectories.

First-principles calculations typically compute the drift mobility $\mu_{\mathrm{d}}$ in zero magnetic field, whereas in experiments a common practice is to obtain the mobility from Hall measurements [46]; the resulting Hall mobility is defined as $\mu_{H}=\sigma_{\mathrm{d}} R_{\mathrm{H}}$, where $\sigma_{\mathrm{d}}$ is the drift conductivity and $R_{\mathrm{H}}$ the Hall coefficient. In Drude theory, $R_{\mathrm{H}}$ evaluates to $1 / n e$ for a carrier concentration $n$ [1], so $\mu_{\mathrm{H}}=\mu_{\mathrm{d}}$. However, when the dependence of the relaxation time on the electronic state is taken into account, $R_{\mathrm{H}}$ deviates from the Drude value by the Hall factor $r=\mu_{\mathrm{H}} / \mu_{\mathrm{d}}$ [24], so the Hall and drift mobilities differ by the Hall factor. For systems with cubic symmetry and $\mathbf{B}$ field in the $z$ direction, the Hall factor is $r=n e \sigma_{x y z}^{(1)} /\left(\sigma_{x x}^{(0)}\right)^{2}[25]$.

\section{B. Computational details}

We apply our approach to $\mathrm{Si}, \mathrm{GaAs}$, and graphene. Their ground state is computed using DFT in the local density approximation, with a plane-wave basis set and normconserving pseudopotentials, using the QUANTUM ESPRESSO package. We use plane-wave kinetic energy cutoffs of $40 \mathrm{Ry}$ for $\mathrm{Si}, 72 \mathrm{Ry}$ for $\mathrm{GaAs}$, and $90 \mathrm{Ry}$ for graphene and relaxed lattice parameters of $5.43 \AA$ for $\mathrm{Si}, 5.56 \AA$ for $\mathrm{GaAs}$, and $2.44 \AA$ for graphene. The phonon dispersions and $e$-ph perturbation potentials on coarse q-point grids are computed with DFPT [51] and the Wannier functions are obtained using WANNIER90 [52]. We use coarse electron k-point and phonon q-point grids of $8 \times 8 \times 8$ for $\mathrm{Si}$ and GaAs and $36 \times 36 \times 1 \mathbf{k}$ and $18 \times 18 \times 1 \mathbf{q}$ points for graphene. We compute and interpolate the $e$-ph matrix elements using our PERTURBO open source package [43]. Calculations with SOC [43,53] employ 

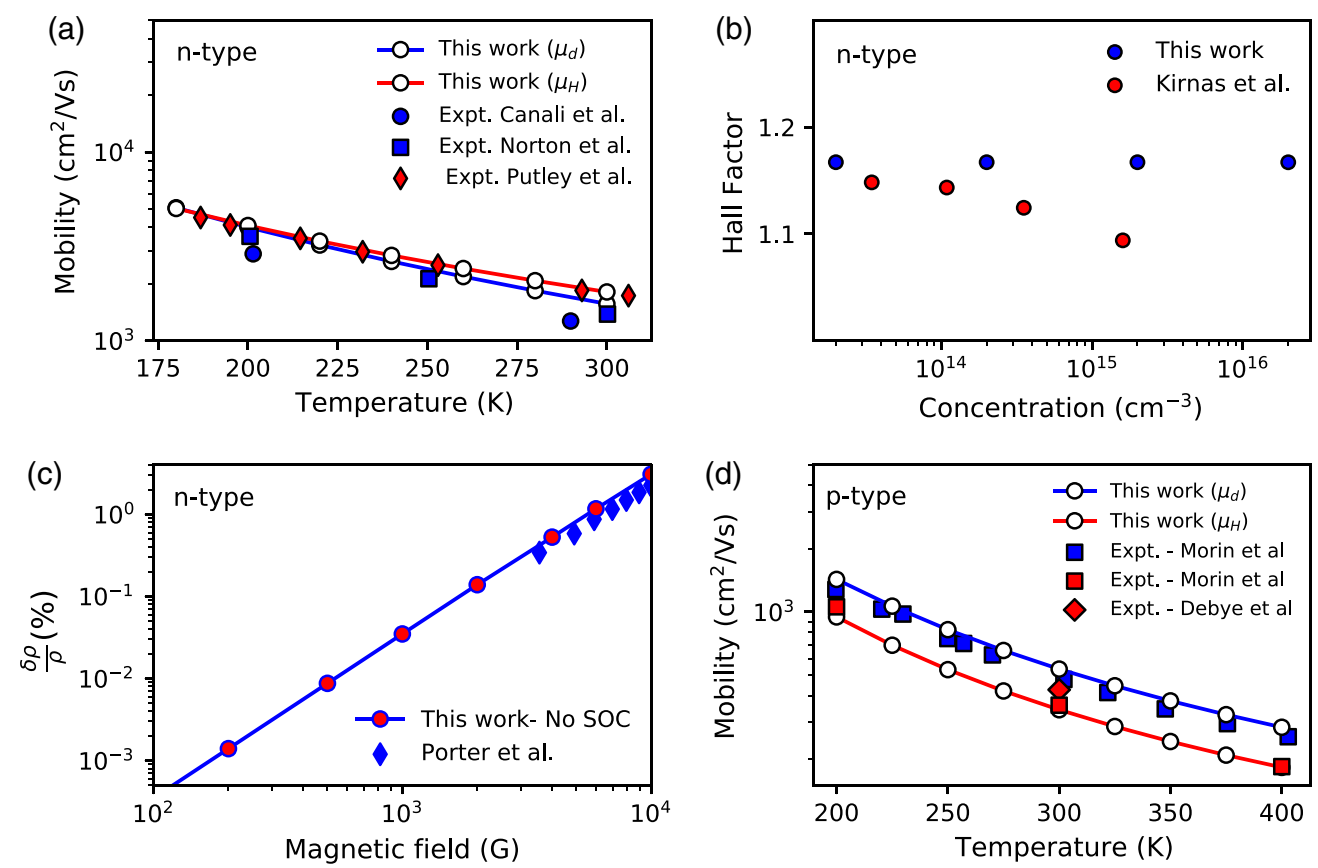

FIG. 1. (a) Drift and Hall mobilities in blue and red, respectively, as a function of temperature in $n$-type silicon (experimental data are from Refs. [12,47-49]). (b) Hall factor at $300 \mathrm{~K}$ as a function of carrier concentration in $n$-type silicon. (c) Transverse MR as a function of magnetic field in $n$-type silicon, compared with experiments from Ref. [8]. (d) Drift and Hall mobilities as a function of temperature in $p$-type silicon (experimental data are taken from Refs. $[9,11,50]$ ).

fully relativistic pseudopotentials. We implement the iterative solution of the BTE in a magnetic field in PERTURBO, and use very fine, equal and uniform $\mathbf{k}$ - and $\mathbf{q}$-point grids (with $140^{3}$ points for $\mathrm{Si}, 650^{3}$ for $\mathrm{GaAs}$ and $1800^{2}$ for graphene) to converge the BTE solutions. The conductivity tensor is obtained via tetrahedron integration [43].

\section{RESULTS}

\section{A. Silicon}

We compute the drift and Hall mobilities, Hall factor, and $\mathrm{MR}$ as a function of temperature for $\mathrm{Si}$ and compare the computed results with experiments. Figure 1(a) shows the Hall and drift electron mobilities in $n$-type silicon. The agreement with experimental data from Refs. [12,47-49] is excellent. As expected for electron carriers, the Hall mobility is greater than the drift mobility at all temperatures. The computed Hall factor, $r=\mu_{\mathrm{H}} / \mu_{\mathrm{d}}$, increases slightly with temperature, as evidenced by higher deviations between $\mu_{\mathrm{H}}$ and $\mu_{\mathrm{d}}$ for higher temperatures.

The Hall factor for electrons is shown in Fig. 1(b) at $300 \mathrm{~K}$ as a function of carrier concentration, which can be tuned in our calculations by changing the chemical potential. At low carrier density, our computed Hall factor is very close to the accepted value of $\sim 1.15$ in $n$-type $\mathrm{Si}$ [7]. The computed Hall factor is within $\sim 10 \%$ of experiment at all carrier concentrations, a noteworthy result for a calculation without adjustable parameters. We attribute the increasing deviation from experiments at higher concentrations to scattering from ionized impurities not taken into account in this paper.
The transverse MR is a common figure of merit for various applications. In Fig. 1(c), we plot the transverse MR as a function of magnetic field for electron carriers in $n$-type $\mathrm{Si}$. The computed MR is in very good agreement with experiments from Ref. [8]. In the low field regime $\mu_{\mathrm{H}} \mathrm{B} \ll 1$, the calculations use a strict convergence threshold on the conductivity, with a change $\Delta \sigma / \sigma<10^{-8}$ between consecutive iterations. Calculations at higher fields $\left(B>2 \times 10^{3} \mathrm{G}\right)$ require relaxing this convergence threshold to $\sim 10^{-4}$. Remaining differences between experiment and theory may be due to various factors, including uncertainty in the experimental temperature and doping concentration, as well as inevitable small deviations from experiment of the computed band structure and phonon dispersions.

Figure 1(d) shows the computed Hall and drift mobilities of hole carriers and compares them with experimental data for $p$-type silicon. The hole mobilities are in very good agreement with data for $p$-type $\mathrm{Si}[9,11,50]$. For hole carriers, correctly, we obtain a behavior opposite to electrons, $\mu_{\mathrm{H}}<\mu_{\mathrm{d}}$ in the entire temperature range and thus a Hall factor $r<1$. Our computed low-field MR coefficient, $\mathrm{MR} / \mathrm{B}^{2}$, is $7.74 \times 10^{5} \mathrm{~cm}^{4} / \mathrm{V}^{2} \mathrm{~s}^{2}$ for holes at $300 \mathrm{~K}$, within $30 \%$ of the measured value of $5.90 \times 10^{5} \mathrm{~cm}^{4} / \mathrm{V}^{2} \mathrm{~s}^{2}$ [54]. For hole carriers, we find that calculations without SOC fail to produce an isotropic conductivity tensor, a key sanity check for Si (for electrons, SOC has only a minor effect). The spurious MR anisotropy for calculations without SOC is due to poor numerical convergence and greater errors in the conductivity tensor, providing further evidence that including SOC in our band structure and $e$-ph calculations $[43,53]$ is key to obtaining accurate magnetotransport for hole 

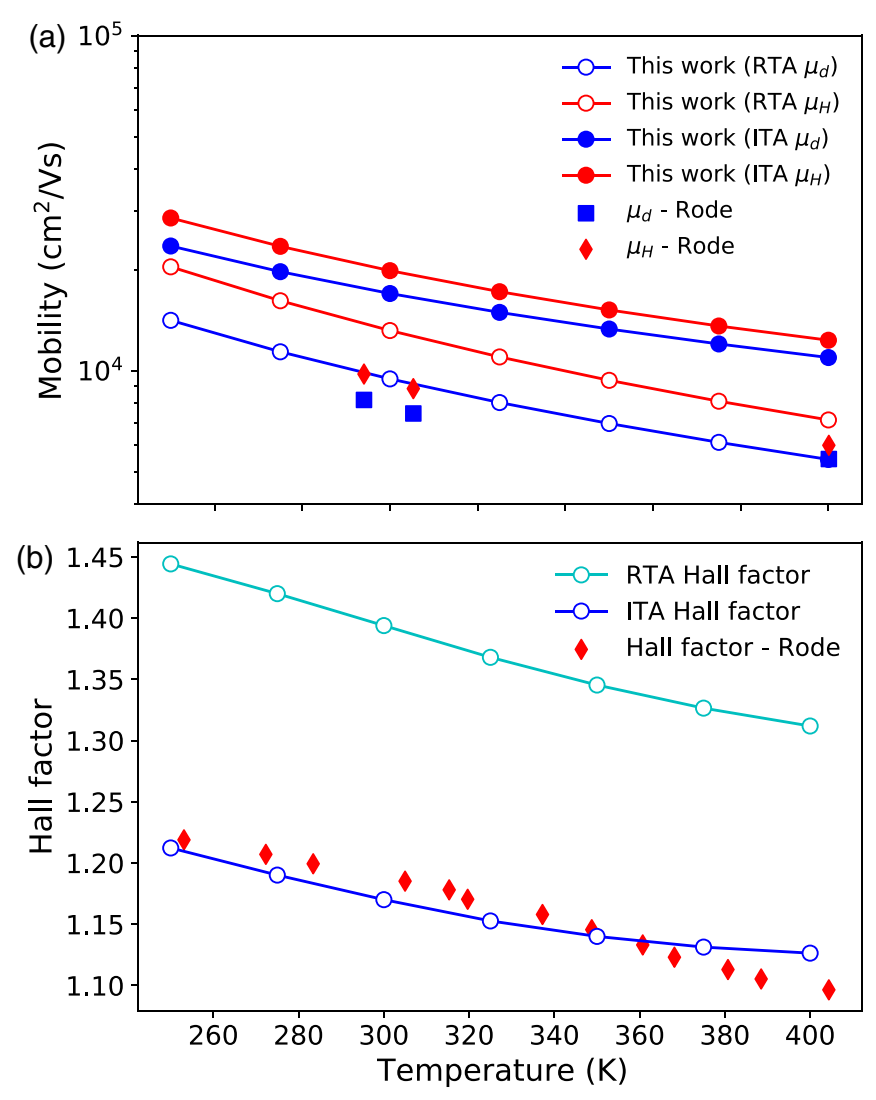

FIG. 2. (a) Drift and Hall mobilities in GaAs as a function of temperature for an electron concentration $n=10^{16} \mathrm{~cm}^{-3}$. (b) Hall factor versus temperature for electrons in GaAs.

carriers. These results show that including SOC makes accurate magnetotransport calculations possible for hole carriers in semiconductors.

\section{B. Gallium arsenide}

The drift mobility has been studied extensively from first principles in GaAs [31,34,37]. Due to its polar character, electrons in GaAs couple strongly with longitudinal optical (LO) phonons through the Fröhlich interaction. We have recently shown that the iterative approach (ITA) to solve the BTE overestimates the mobility and that including electron-two-phonon $(e-2 \mathrm{ph})$ scattering processes significantly improves the result; the RTA also gives a mobility in agreement with experiments [31], but due to compensation of errors [34]. We find that the same trends also hold for the Hall mobility. Figure 2(a) shows the drift and Hall mobilities for electrons in GaAs as a function of temperature. The experimental Hall mobility shown for comparison is obtained from Ref. [13].

The ITA overestimates both the drift and Hall mobilities, by a factor of $\sim 2$ at $300 \mathrm{~K}$, while the RTA is in better agreement with experiments due to error compensation [34]. The Hall factor $r=\mu_{\mathrm{H}} / \mu_{\mathrm{d}}$ for both approaches is correctly greater than 1, but the Hall factor for ITA is much closer to the measured data [Fig. 2(b)]. Although each of the Hall and drift mobilities are overestimated in the ITA, their ratio is predicted accurately; we cannot establish whether this result is a coincidence or due to cancellation of effects from $e-2 \mathrm{ph}$
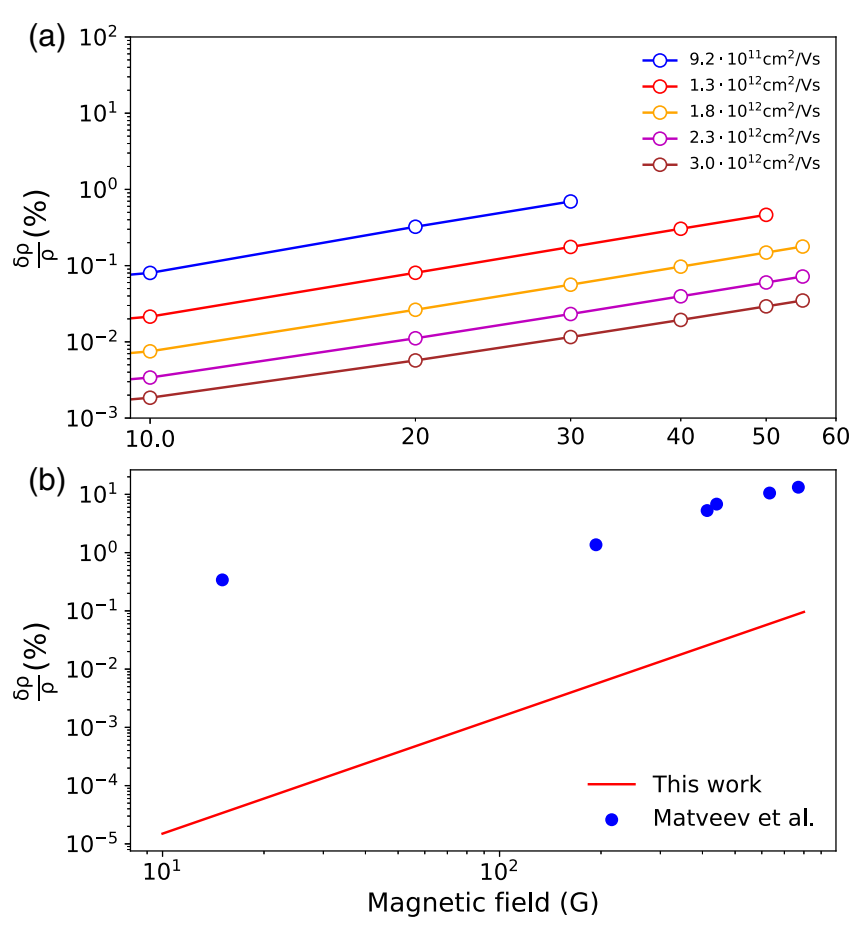

FIG. 3. (a) Transverse MR in graphene versus magnetic field for various hole carrier concentrations at $300 \mathrm{~K}$. (b) Comparison of the calculated MR for a hole concentration of $p=2.46 \times 10^{13} \mathrm{~cm}^{-2}$ with experimental data for $p=(2.2-4.2) \times 10^{13} \mathrm{~cm}^{-2}$, taken from Ref. [16].

processes in the ratio. Overall, these trends show that for polar semiconductors first-principles magnetotransport calculations have an accuracy similar to calculations without magnetic field.

\section{Graphene}

Similar to other semimetals [1], graphene exhibits a relatively large MR, with reported values of $20-50 \%$ at room temperature and even greater at lower temperatures [17]. We discuss the MR in graphene for hole carriers but the MR values for electrons are similar. The accuracy of our settings is checked by calculating the drift mobility at $300 \mathrm{~K}$; we obtain a value of $\sim 160000 \mathrm{~cm}^{2} /$ Vs consistent with experiments in suspended graphene [55].

Figure 3(a) shows the computed MR in graphene at $300 \mathrm{~K}$. We find that the MR depends strongly on carrier concentration- $\mathrm{a}$ doubling of concentration from $\sim 1.5$ to $3 \times 10^{12} \mathrm{~cm}^{2} /$ Vs decreases the MR by an order of magnitude. This situation makes comparison with experiments difficult [Fig. 3(b)] as the reported carrier concentration usually does not take into account the Hall factor (we find $r=1.45$ for $n=1.2 \times 10^{12} \mathrm{~cm}^{-3}$, consistent with recent work [41]). As by definition $n=r /\left(e R_{\mathrm{H}}\right)$, carrier concentrations from Hall measurements are inaccurate unless the Hall factor is taken into account. In addition, most graphene samples are measured on substrates, often causing a reduction in the mobility. Accordingly, experimental values of the mobility and MR vary over a wide range $[17,18,56]$. This variability in the ex- 

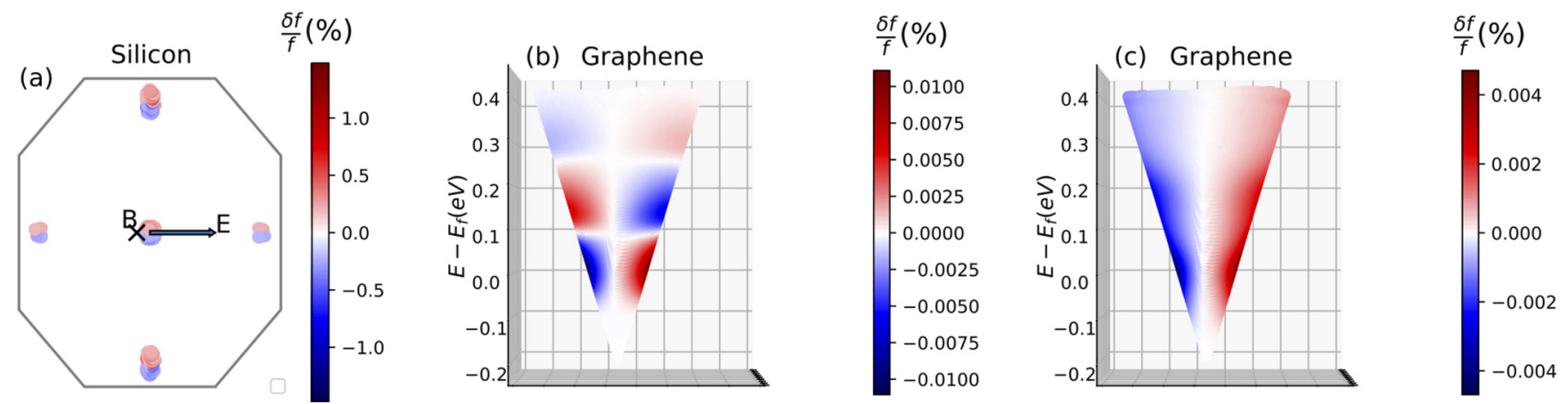

FIG. 4. (a) Projection of the occupation changes $\delta f / f$ onto the $k_{z}=0$ plane in Si. (b) Occupation change $\delta f / f$ in graphene near the Dirac cone with contributions from all phonons. (c) The same result as in (b) for graphene but with scattering from the highest-energy optical phonon branch excluded from the transport calculation.

perimental results can at least partially explain the discrepancy between the calculated and measured MR in Fig. 3(b).

Analysis of the electron occupations (see below) reveals that taking into account backscattering by iteratively solving the full BTE (as opposed to using the RTA) is essential in graphene, and that the RTA fails to capture the correct electronic occupations at steady state. This key role of backscattering in magnetotransport mimics trends found for thermal transport in graphene [57].

\section{Steady-state occupations}

To conclude our analysis, we study the electron occupations at steady state, focusing on their change due to the magnetic field for a constant electric field. We define this relative occupation change as $\delta f / f=\left[f_{n \mathbf{k}}(\mathbf{E}, \mathbf{B})-\right.$ $\left.f_{n \mathbf{k}}(\mathbf{E}, 0)\right] / f_{n \mathbf{k}}(\mathbf{E}, 0)$ and plot it in momentum space for $\mathrm{Si}$ and graphene. In the results for $\mathrm{Si}$, shown in Fig. 4(a), the occupation change projected on the $k_{z}=0$ plane clearly shows the effect of the Lorentz force, whereby the electrons deviate in the $\mathbf{E} \times \mathbf{B}$ direction near the six conduction band minima (the occupations at the zone center are projections of the two band minima along the $k_{z}$ axis). As expected, the electrons are deflected in momentum space due to the magnetic field, an important sanity check for our numerical implementation.

The results for graphene, shown in Fig. 4(b), are more interesting. Similar to Si, the occupations near the Dirac cone are also changed by the Lorentz force. However, electrons in graphene couple strongly with LO phonons with momentum near $\Gamma$ and transverse optical (TO) phonons with momentum near $K$ [58], which mediate intra- and intervalley electronic processes, respectively. As a result, optical phonon absorption generates a steplike pattern in the occupation changes, with $160-200 \mathrm{meV}$ spacing equal to the LO and TO phonon energies [58]. The disappearance of the alternating patches on removing scattering from the highest optical branch from the transport calculation [Fig. 4(c)] provides concrete evidence for the dominant optical phonon backscattering in graphene. The RTA completely misses this trend and gives occupation changes without a steplike pattern, as shown in Fig. 4(c). While in graphene the magnetotransport RTA results are in fairly good agreement with the full solution of the BTE, which correctly includes backscattering, our results show that the
RTA fails to capture important features of magnetotransport in graphene.

\section{CONCLUSION}

We have shown calculations of magnetotransport that can accurately predict the Hall mobility, Hall factor, and MR in $\mathrm{Si}$ and GaAs. Our results for graphene leave room for improvements and call for stricter protocols for magnetotransport measurements in 2D materials. Analysis of the steady-state occupations in graphene highlights a key strength of first-principles calculations - they can capture the competition between mode-dependent $e$-ph scattering and the effect of the Lorentz force in momentum space, shedding light on the microscopic mechanisms governing magnetotransport. With calculations on materials with tens of atoms in the unit cell readily available [43], an extension of these results to other semiconductors and 2D materials appears straightforward. The current formalism can be easily extended to include the Berry curvature, for example, to study magnetotransport in topological semimetals and shed light on the origin of their unconventional MR. Our method is general and can be applied to a wide range of materials, including semimetals such as bismuth and graphite with large MR and topological semimetals with strong SOC and unconventional magnetotransport. As our approach allows us to calculate the full resistivity tensor, it will be interesting to apply it in the future to interpret experimental angular MR diagrams. The magnetotransport calculations shown in this paper will be made available in our PERTURBO code, thus greatly expanding the reach of firstprinciples transport studies and connecting them more deeply with transport experiments, which are often carried out in magnetic fields.

\section{ACKNOWLEDGMENTS}

This work was supported by the National Science Foundation under Grant No. DMR-1750613. J.-J.Z. acknowledges partial support from the Joint Center for Artificial Photosynthesis, a DOE Energy Innovation Hub, as follows: The development of some computational methods employed in this work was supported through the Office of Science of the US Department of Energy under Award No. DE-SC0004993. This research used resources of the National Energy Research 
Scientific Computing Center (NERSC), a U.S. Department of Energy Office of Science User Facility located at Lawrence
Berkeley National Laboratory, operated under Contract No. DE-AC02-05CH11231.
[1] J. M. Ziman, Electrons and Phonons: The Theory of Transport Phenomena in Solids (Oxford University Press, Oxford, 2001), p. 483.

[2] A. B. Pippard, Magnetoresistance in Metals (Cambridge University Press, Cambridge, 1989), Vol. 2.

[3] S. Parkin, Giant magnetoresistance in magnetic nanostructures, Annu. Rev. Mater. Sci. 25, 357 (1995).

[4] A. Ramirez, Colossal magnetoresistance, J. Phys. Condens. Matter 9, 8171 (1997).

[5] J. E. Lenz, A review of magnetic sensors, Proc. IEEE 78, 973 (1990).

[6] J. M. Daughton, GMR applications, J. Magn. Magn. Mater. 192, 334 (1999).

[7] I. G. Kirnas, P. M. Kurilo, P. G. Litovchenko, V. S. Lutsyak, and V. M. Nitsovich, Concentration dependence of the Hall factor in n-type silicon, Phys. Status Solidi (a) 23, K123 (1974).

[8] N. A. Porter and C. H. Marrows, Dependence of magnetoresistance on dopant density in phosphorous doped silicon, J. Appl. Phys. 109, 07C703 (2011).

[9] F. J. Morin and J. P. Maita, Electrical properties of silicon containing arsenic and boron, Phys. Rev. 96, 28 (1954).

[10] W. E. Krag, Galvanomagnetic effects in $n$-type silicon, Phys. Rev. 118, 435 (1960).

[11] P. P. Debye and T. Kohane, Hall mobility of electrons and holes in silicon, Phys. Rev. 94, 724 (1954).

[12] E. H. Putley and W. H. Mitchell, The electrical conductivity and Hall effect of silicon, Proc. Phys. Soc. 72, 193 (1958).

[13] D. L. Rode, Semiconductors and Semimetals (Academic Press, New York, 1975); D. L. Rode and S. Knight, Electron transport in GaAs, Phys. Rev. B 3, 2534 (1971).

[14] G. Stillman, C. Wolfe, and J. Dimmock, Hall coefficient factor for polar mode scattering in n-type GaAs, J. Phys. Chem. Solids 31, 1199 (1970).

[15] J. S. Blakemore, Semiconducting and other major properties of gallium arsenide, J. Appl. Phys. 53, R123 (1982).

[16] V. Matveev, V. Levashov, O. Kononenko, and V. Volkov, Large positive magnetoresistance of graphene at room temperature in magnetic fields up to 0.5t, Scr. Mater. 147, 37 (2018).

[17] K. Gopinadhan, Y. J. Shin, I. Yudhistira, J. Niu, and H. Yang, Giant magnetoresistance in single-layer graphene flakes with a gate-voltage-tunable weak antilocalization, Phys. Rev. B 88, 195429 (2013).

[18] W. J. Wang, K. H. Gao, Z. Q. Li, T. Lin, J. Li, C. Yu, and Z. H. Feng, Classical linear magnetoresistance in epitaxial graphene on SiC, Appl. Phys. Lett. 105, 182102 (2014).

[19] M. N. Ali, J. Xiong, S. Flynn, J. Tao, Q. D. Gibson, L. M. Schoop, T. Liang, N. Haldolaarachchige, M. Hirschberger, N. P. Ong, and R. J. Cava, Large, non-saturating magnetoresistance in $\mathrm{WTe}_{2}$, Nature 514, 205 (2014).

[20] J. Xiong, S. K. Kushwaha, T. Liang, J. W. Krizan, M. Hirschberger, W. Wang, R. J. Cava, and N. P. Ong, Evidence for the chiral anomaly in the Dirac semimetal $\mathrm{Na}_{3} \mathrm{Bi}$, Science 350, 413 (2015).
[21] C.-Z. Li, L.-X. Wang, H. Liu, J. Wang, Z.-M. Liao, and D.-P. $\mathrm{Yu}$, Giant negative magnetoresistance induced by the chiral anomaly in individual $\mathrm{Cd}_{3} \mathrm{As}_{2}$ nanowires, Nat. Commun. 6, 10137 (2015).

[22] N. P. Armitage, E. J. Mele, and A. Vishwanath, Weyl and Dirac semimetals in three-dimensional solids, Rev. Mod. Phys. 90, 015001 (2018).

[23] J.-P. Jan, Galvamomagnetic and thermomagnetic effects in metals, in Solid State Physics (Elsevier, New York, 1957), Vol. 5, pp. 1-96.

[24] J. Lin, S. Li, L. Linares, and K. Teng, Theoretical analysis of Hall factor and Hall mobility in p-type silicon, Solid State Electron. 24, 827 (1981).

[25] L. Reggiani, D. Waechter, and S. Zukotynski, Hall-coefficient factor and inverse valence-band parameters of holes in natural diamond, Phys. Rev. B 28, 3550 (1983).

[26] B. F. Lewis and E. H. Sondheimer, The theory of the magnetoresistance effects in polar semi-conductors, Proc. R. Soc. Lond. A 227, 241 (1955).

[27] F. Szmulowicz, Calculation of optical- and acoustic-phononlimited conductivity and Hall mobilities for $p$-type silicon and germanium, Phys. Rev. B 28, 5943 (1983).

[28] F. Szmulowicz and F. L. Madarasz, Deformation-potentialtheory calculation of the acoustic-phonon-limited conductivity and Hall mobilities for $p$-type silicon, Phys. Rev. B 27, 2605 (1983).

[29] R. M. Martin, Electronic Structure: Basic Theory and Practical Methods (Cambridge University Press, Cambridge, 2004).

[30] S. Baroni, S. de Gironcoli, A. Dal Corso, and P. Giannozzi, Phonons and related crystal properties from density-functional perturbation theory, Rev. Mod. Phys. 73, 515 (2001).

[31] J.-J. Zhou and M. Bernardi, Ab initio electron mobility and polar phonon scattering in GaAs, Phys. Rev. B 94, 201201(R) (2016).

[32] V. A. Jhalani, J.-J. Zhou, and M. Bernardi, Ultrafast hot carrier dynamics in $\mathrm{GaN}$ and its impact on the efficiency droop, Nano Lett. 17, 5012 (2017).

[33] N.-E. Lee, J.-J. Zhou, L. A. Agapito, and M. Bernardi, Charge transport in organic molecular semiconductors from first principles: The bandlike hole mobility in a naphthalene crystal, Phys. Rev. B 97, 115203 (2018).

[34] N.-E. Lee, J.-J. Zhou, H.-Y. Chen, and M. Bernardi, Ab initio electron-two-phonon scattering in GaAs from next-to-leading order perturbation theory, Nat. Commun. 11, 1607 (2020).

[35] J. Park, J.-J. Zhou, V. A. Jhalani, C. E. Dreyer, and M. Bernardi, Long-range quadrupole electron-phonon interaction from first principles, Phys. Rev. B 102, 125203 (2020).

[36] W. Li, Electrical transport limited by electron-phonon coupling from Boltzmann transport equation: An ab initio study of $\mathrm{Si}$, Al, and $\mathrm{MoS}_{2}$, Phys. Rev. B 92, 075405 (2015).

[37] T.-H. Liu, J. Zhou, B. Liao, D. J. Singh, and G. Chen, First-principles mode-by-mode analysis for electron-phonon scattering channels and mean free path spectra in GaAs, Phys. Rev. B 95, 075206 (2017). 
[38] J. Ma, A. S. Nissimagoudar, and W. Li, First-principles study of electron and hole mobilities of Si and GaAs, Phys. Rev. B 97, 045201 (2018).

[39] T. Sohier, D. Campi, N. Marzari, and M. Gibertini, Mobility of two-dimensional materials from first principles in an accurate and automated framework, Phys. Rev. Mater. 2, 114010 (2018).

[40] F. Macheda and N. Bonini, Magnetotransport phenomena in p-doped diamond from first principles, Phys. Rev. B 98, 201201(R) (2018).

[41] F. Macheda, S. Poncé, F. Giustino, and N. Bonini, Theory and computation of Hall scattering factor in graphene, Nano Lett. 20, 8861 (2020).

[42] S. N. Zhang, Q. S. Wu, Liu, and O. V. Yazyev, Magnetoresistance from Fermi surface topology, Phys. Rev. B 99, 035142 (2019).

[43] J.-J. Zhou, J. Park, I.-T. Lu, I. Maliyov, X. Tong, and M. Bernardi, PERTURBO: A software package for ab initio electronphonon interactions, charge transport and ultrafast dynamics, Comput. Phys. Commun., 107970 (2021).

[44] A. A. Mostofi, J. R. Yates, Y.-S. Lee, I. Souza, D. Vanderbilt, and N. Marzari, Wannier90: A tool for obtaining maximallylocalised wannier functions, Comput. Phys. Commun. 178, 685 (2008).

[45] W. Jones and N. March, Theoretical Solid State Physics (Wiley, London, 1973).

[46] S. M. Sze and K. K. Ng, Physics of Semiconductor Devices (John Wiley and Sons, Hoboken, NJ, 2006).

[47] C. Canali, C. Jacoboni, F. Nava, G. Ottaviani, and A. AlberigiQuaranta, Electron drift velocity in silicon, Phys. Rev. B 12, 2265 (1975).

[48] R. A. Logan and A. J. Peters, Impurity effects upon mobility in silicon, J. Appl. Phys. 31, 122 (1960).

[49] P. Norton, T. Braggins, and H. Levinstein, Impurity and lattice scattering parameters as determined from Hall and mobility analysis in $n$-type silicon, Phys. Rev. B 8, 5632 (1973).
[50] J. Dorkel and P. Leturcq, Carrier mobilities in silicon semiempirically related to temperature, doping and injection level, Solid State Electron. 24, 821 (1981).

[51] P. Giannozzi, S. Baroni, N. Bonini, M. Calandra, R. Car, C. Cavazzoni, D. Ceresoli, G. L. Chiarotti, M. Cococcioni, I. Dabo, A. D. Corso, S. de Gironcoli, S. Fabris, G. Fratesi, R. Gebauer, U. Gerstmann, C. Gougoussis, A. Kokalj, M. Lazzeri, L. Martin-Samos, N. Marzari, F. Mauri, R. Mazzarello, S. Paolini, A. Pasquarello, L. Paulatto, C. Sbraccia, S. Scandolo, G. Sclauzero, A. P. Seitsonen, A. Smogunov, P. Umari, and R. M. Wentzcovitch, QUANTUM ESPRESSO: A modular and open-source software project for quantum simulations of materials, J. Phys. Condens. Matter 21, 395502 (2009).

[52] A. A. Mostofi, J. R. Yates, G. Pizzi, Y.-S. Lee, I. Souza, D. Vanderbilt, and N. Marzari, An updated version of WANNIER90: A tool for obtaining maximally-localised Wannier functions, Comput. Phys. Commun. 185, 2309 (2014).

[53] J. Park, J.-J. Zhou, and M. Bernardi, Spin-phonon relaxation times in centrosymmetric materials from first principles, Phys. Rev. B 101, 045202 (2020).

[54] D. Long and J. Myers, Weak-field magnetoresistance in p-type silicon, Phys. Rev. 109, 1098 (1958).

[55] K. Bolotin, K. Sikes, Z. Jiang, M. Klima, G. Fudenberg, J. Hone, P. Kim, and H. Stormer, Ultrahigh electron mobility in suspended graphene, Solid State Commun. 146, 351 (2008).

[56] M. Rein, N. Richter, K. Parvez, X. Feng, H. Sachdev, M. Kläui, and K. Müllen, Magnetoresistance and charge transport in graphene governed by nitrogen dopants, ACS Nano 9, 1360 (2015).

[57] G. Fugallo, A. Cepellotti, L. Paulatto, M. Lazzeri, N. Marzari, and F. Mauri, Thermal conductivity of graphene and graphite: Collective excitations and mean free paths, Nano Lett. 14, 6109 (2014).

[58] X. Tong and M. Bernardi, Toward precise simulations of the coupled ultrafast dynamics of electrons and atomic vibrations in materials, arXiv:2009.07958. 